\title{
Mécanismes de la cancérogenèse par les rayonnements ionisants
}

\author{
J. COPPEY *
}

(Manuscrit reçu le 20 septembre 1989)

RÉSUMÉ Les radiations ionisantes induisent de multiples dommages par les espèces radicalaires très réactives qu'elles génèrent au sein des tissus dans lesquels elles sont absorbées. Certains de ces dommages : cassures ou brèches dans I'ADN et peroxydes membranaires peuvent enclencher des processus donnant naissance à plusieurs types de cancers. Les plus fréquents sont des leucémies, des sarcomes ou des tumeurs de la thyroïde. Plus spécifiquement, différents résultats tirés d'observations cytogénétiques dans les radioleucémies, d'études de désordres génétiques confẻrant une susceptibilité accrue aux sarcomes radioinduits, et de l'analyse des propriétés transformantes des rayonnements en cellules cultivées, indiquent que la tumorigenèse radioprovoquée résulte essentiellement d'une perte stable de gènes dont le produit exerce des fonctions suppressives vis-à-vis de la transformation ou de l'expansion clonale des cellules transformées. Cette perte peut être directement créée ou provenir d'une réparation (par recombinaison) infidèle des cassures ou des brèches de I'ADN. Les fonctions suppressives pourraient impliquer des activités antiprotéasiques.

ABSTRACT Ionizing radiations induce multipe damages via the reactive radical species they create inside the tissues where they are absorbed. Some damages such as breaks or gaps in DNA and membrane peroxides can trigger processes leading to several types of cancers : leukemias, sarcomas or thyroid tumors. More specifically, different results arising from cytogenetical observations in radioleukemia, from studies on genetical disorders confering high susceptibility to radiation induced sarcomas and from analyses of the transforming properties of radiations in culfured cells indicate that radiation induced tumorigenesis mainly results from a stable loss of genes, the product of which exerts suppressive functions towards transformation or clonal growth of transformed cells. The gene loss can be directly provoked or arise from an unfaithfull recombinational repair of the DNA breaks or gaps. The suppressive functions could involve antiprotease activities.

La croissance, la différenciation et le vieillissement harmonieux des différents organes des vertébrés reposent sur le fonctionnement normal de chacune des cellules qui les composent. Ce qui implique la stabilité de la structure primaire de l'ADN, réservoir d'information potentielle de dizaines de milliers de gènes : toute mutation ponctuelle ou délétion peut l'altérer. Ce qui implique également la plasticité de la structure spatiale de l'ADN et des protéines qui lui sont associées, plasticité dont dépend la

* Institut Curie, section de Biologie, 26, rue d'Ulm, 75231 Paris Cedex 05. 
régulation de l'expression génétique. Cette plasticité résulte de transitions possibles entre conformations diverses, lesquelles mettent en jeu de nombreux facteurs : degré de superenroulement et taux de méthylation de l'ADN au sein de boucles de 5 à 100 kilobases, correspondant à des unités de transcription [9], nature (réversible ou non) des interactions entre ADN et protéines de la matrice ou protéines de structure (histone $\mathrm{H} 1$ en particulier) [9], entre ADN et protéines de régulation, activateurs ou répresseurs $[3,35]$, voire même entre protéines de régulation [3]. Des effets modulateurs sont créés en permanence par des facteurs extrinsèques : signaux transductionnels, mettant en œuvre le métabolisme des phospholipides membranaires [4]. Ces signaux enclenchés par interaction entre facteurs de croissance (hormones, peptides divers dont les neuropeptides) et leurs récepteurs génèrent des flux ioniques. C'est la coordination de cet ensemble qui règle l'expression génétique au cours du développement tissulaire [35-36].

Les espèces radicalaires induites par les rayonnements ionisants (RI) sont sources de dommages à tous les niveaux précédemment mentionnés. On peut considérer, cependant, que pour des doses critiques en terme de tumorigenèse (de l'ordre de quelques grays), les cassures de l'ADN et les peroxydations des phospholipides des membranes représentent les dommages les plus conséquents. Des données de l'épidémiologie et de l'expérimentation animale nous apprennent qu'au-delà d'un seuil de dose, assez variable selon le cas [17], les RI ont pour effet d'augmenter l'incidence de cancers, principalement des leucémies, des sarcomes et des cancers de la thyroide. Par ailleurs, une simple exposition aux RI peut augmenter l'incidence de cancers liés à un contact avec une substance cancérogène. Le contact peut avoir lieu jusqu'à 10-20 ans après l'irradiation (données exposées dans [1]). Ainsi, les RI peuvent être des facteurs de cancérogenèse ou de cocancérogenèse.

Les résultats d'expériences chez la drosophile, le poisson et les petits rongeurs montrent qu'il existe des déterminants génétiques qui confèrent une susceptibilité accrue vis-à-vis des cancers, parmi lesquels ceux favorisés par les RI. Cette susceptibilité semble être due à des mutations dans des gènes récessifs (ou à leur perte) : ces gènes codent pour des fonctions suppressives de la tumorigenèse [12]. Une susceptibilité de même nature existe chez l'homme. C'est le cas du rétinoblastome bilatéral, désordre héréditaire résultant du manque d'un gène (Rb) porté par le chromosome 13. La perte du gène n'est visible par cytogénétique que dans $10 \%$ des cas. Les enfants $\mathrm{Rb}^{+} / \mathrm{Rb}^{-}$à la naissance développent très tôt des tumeurs de rétine dont le génotype est $\mathrm{Rb}^{-} / \mathrm{Rb}^{-}$. A la suite du traitement de ces tumeurs par radiothérapie, des ostéosarcomes de génotype également $R b^{-} / R b^{-}$apparaissent très fréquemment. Le gène $\mathrm{Rb}$ a été récemment isolé (réf. dans [33]), et sa structure génomique a été caractérisée [14]. Introduit dans des cellules tumorales, il bloque leur croissance in vivo [33]. Le gène $\mathrm{Rb}$ est sous forme non active dans certaines tumeurs du sein [18]. Ce gène représente le prototype d'un antioncogène humain. Cependant, la perte du gène $\mathrm{Rb}$ dans les tumeurs de rétine et les ostéosarcomes secondaires s'accompagne constamment d'aberrations d'un type particulier (réf. dans [28]), ce qui conduit à 
supposer que la très probable activation d'un oncogène s'ajoute à la perte de l'antioncogène dans la genèse de ces tumeurs. Une rupture d'équilibre avec surexpression d'un oncogène doublée d'une non-expression d'antioncogène pourrait, d'ailleurs, être de règle dans de nombreux processus tumorigènes [16]. Des données cytogénétiques confortent l'idée selon laquelle le pouvoir cancérogène des RI implique une perte d'information génétique particulière. Ainsi, dans les leucémies radioprovoquées comme dans celles provoquées par des drogues radiomimétiques, on relève des délétions du bras long des chromosomes 5 ou 7 , ou du bras court du 17 [23 et 1, p. 14].

Les effets de l'irradiation de cellules cultivées in vitro nous fournissent des informations sur les mécanismes de la radiocancérogenèse. Les cellules embryonnaires humaines (dont la durée de vie en culture est limitée) ne sont pas transformables par les RI [15]. Cependant, des souspopulations de cellules porteuses de réarrangements chromosomiques d'un type donné émergent peu à peu des cultures irradiées [15]. L'émergence de ces sous-populations pourrait représenter une étaje d'initiation de la transformation. Ceci parce que la quasi-totalité des tumeurs humaines présentent des réarrangements réalisant des translocations stables [16]. Des événements de recombinaison illégitime enclenchés par des cassures double chaine de l'ADN (voir plus loin) sont à l'origine des réarrangements. Les cellules embryonnaires de rongeur immortalisées sont, par contre, aisément transformables par les RI : la transformation est induite au-delà d'un seuil de dose de l'ordre de 0,5 Gy (voir [4] pour revue). Des applications répétées de promoteurs comme les esters actifs de phorbol [20] ou la surexpression après transfection d'un protooncogène (c-myc) dont le produit exerce des effets promoteurs [31] font fortement augmenter le taux de transformation après irradiation. II n'y a plus de seuil de dose dans ces conditions.

Certains des mécanismes de la transformation par les RI commencent à être saisis. Les RI créent des cassures simple chaîne et, en proportion plus petite, des cassures double chaîne, voire des microdélétions dans l'ADN $[11,14]$. Lorsqu'elles ne sont pas réparées, les cassures double chaîne et les délétions constituent des dommages létaux [27]. Une fraction de ces lésions, de par leur nature chimique [14], ne sont pas réparables par religation. Elles le sont seulement par recombinaison homologue ou illégitime.

- Homologue : la séquence intacte de la chromatide-sœur, ou celle portée par l'allèle correspondant, sert de matrice et un échange d'ADN catalysé par un complexe polyenzymatique a lieu [8]. Ce complexe a pu être isolé fonctionnel hors du noyau [21]. Si la recombinaison homologue se déroule d'une façon imparfaite, elle peut générer des délétions.

- Illégitime : de courtes longueurs d'homologie bordant les cassures sont requises pour ce type de recombinaison qui génère des translocations de gènes. De la recombinaison illégitime semble avoir eu lieu dans certaines tumeurs du système hématopolétique où l'on trouve le protooncogène c-myc juxtaposé à une séquence hautement activatrice de 
gènes d'lg [16]. On trouve également des translocations d'un type particulier dans les tumeurs hématopoiétiques de sujets atteints d'ataxie télangectasie (AT), désordre conférant une sensibilité accrue à l'effet létal des RI [25]. Mais la survenue de tumeurs hématopoiétiques chez les sujets AT n'a jamais, jusqu'ici, été liée à un facteur d'irradiation externe. De plus, la nature exacte de la déficience conférant une radiosensibilité extrême des cellules AT demeure inconnue [25], bien que la réparation " imparfaite " des cassures double-chaîne dans ces cellules irradiées [6] puisse rendre compte de cette radiosensibilité extrême.

Un fait intéressant a été observé dans les cellules irradiées : la fréquence d'événements de recombinaison est accrue et cet état persiste au cours des divisions ultérieures (voir [7] pour discussion). Cette observation mérite une étude approfondie dans la mesure où elle peut rendre compte de certains des processus de la tumorigenèse, tels que l'adaptation par expression de gènes appropriés et l'expansion clonale des cellules transformées.

La désintégration de radionucléotides incorporés dans l'ADN nucléaire par voie métabolique : ${ }^{3} \mathrm{H}$ dans la thymidine ou ${ }^{125} \mathrm{l}$ sous forme de 5-iododéoxyuridine provoque la transformation des cellules embryonnaires immortalisées : pour le même nombre de désintégrations par cellule, le taux de transformation est beaucoup plus grand avec ${ }^{125}$ I qu'avec ${ }^{3} \mathrm{H}$ [19]. Or, la désintégration de ${ }^{125} 1$ provoque des délétions étendues, de dizaines voire de centaines de nucléotides [24], alors que celle de ${ }^{3} \mathrm{H}$ provoque des cassures de l'ADN et de petites délétions en proportion modérée (réf. dans [11]). Certes, l'efficacité létale de ${ }^{125}$ l est très supérieure à celle de ${ }^{3} \mathrm{H}$, elle-même plus élevée que celle des RI d'origine exogène [19]. II n'en demeure pas moins qu'à des niveaux de survie voisins de $100 \%$, les efficacités relatives de transformation se présentent ainsi : ${ }^{125} \mathrm{I} \gg{ }^{3} \mathrm{H}>\mathrm{RI}(19)$. Ceci conforte la possibilité selon laquelle le pouvoir transformant des RI serait lié à une perte d'information génétique.

Reste à déterminer si cette information concerne des gènes à fonction suppressive de la transformation maligne $[16,28]$ ou d'autres portés par les cellules normales voisinant les cellules transformées et qui exerceraient un effet de contrôle sur leur croissance. Ces deux possibilités ne sont pas mutuellement exclusives. La seconde possibilité signifie que le produit de gènes exprimés dans les cellules normales diffuse et inhibe l'expansion clonale des cellules transformées. C'est bien ce qui existe dans le cas des cellules transformées par les rayonnements ultraviolets courts : les cellules normales inhibent leur croissance clonale et cet effet est aboli par application de promoteurs [13]. La mise en œuvre de cet effet inhibiteur semble impliquer l'existence de communications entre cellules normales et transformées [26]. L'intégrité fonctionnelle des membranes cellulaires et du métabolisme des phospholipides les composant [4] serait ainsi requise. En ce sens, les peroxydes de phospholipides induits par les RI peuvent représenter des éléments déterminants de la transformation par les RI. Mais cette possibilité n'a pas été explorée. 
Quelle peut être la nature des fonctions des produits de gènes suppresseurs ? Le fait que des antiprotéases bloquent la transformation cellulaire par les R1 [20] fournit un élément de réponse. Deux possibilités sont, en effet, envisageables : ou bien le mécanisme de transformation cellulaire nécessite des événements de protéolyse, ou bien l'expansion clonale des cellules transformées se réalise grâce à une protéolyse locale. Des données diverses plaident en faveur de l'une ou de l'autre possibilité. Concernant la première, des protéases cytoplasmiques (produits d'oncogènes ?) ont été isolées et leur effet a été précisé : il s'agit de l'activation de la réplication de l'ADN nucléaire [32]. Ce pouvoir d'activation peut être neutralisé par des glycoprotéines (produits d'antioncogènes) qui s'accumulent dans la membrane cytoplasmique soit de manière réversible dans les cellules embryonnaires ou celles provenant de sujet jeune, soit de manière irréversible au cours du vieillissement cellulaire [22,32]. Notons que les peroxydations membranaires réalisées par les RI peuvent donner naissance à des pontages entre phospholipides et protéines [34], glycoprotéines entre autres. Concernant la seconde possibilité, on a montré qu'il existe des dégradations protéolytiques en cascade enclenchées par une protéase-clef, l'activateur du plasminogène, lequel est secrété constitutivement par les cellules tumorales. Ces dégradations permettent la migration locale de ces cellules par lyse du gel de collagène les emprisonnant, leur passage à travers les parois ou les couches de cellules endothéliales et, en conséquence, leur greffe à distance [29]. Les cellules normales, fibroblastiques et épithéliales sécrètent par réaction des antiprotéases freinant localement, voire contrecarrant, les activités protéolytiques [29].

Un déséquilibre de cette situation peut être provoqué par des facteurs externes dont les RI. II est possible que le pouvoir tumorigène des $\mathrm{RI}$ soit, en partie, provoqué par un déséquilibre de cette nature, ce qui n'a pas jusqu'ici été étudié.

\section{Conclusion}

Les protooncogènes, gènes gouvernant la croissance et la différenciation tissulaire [2] ont été isolés par génétique moléculaire. L'expression de ces gènes est normalement modulée par le jeu d'activations et de répressions. Leur dérégulation représente une étape déterminante de la transformation maligne lorsqu'elle survient à un certain niveau de différenciation cellulaire [16]. La possibilité d'expansion clonale puis d'essaimage à distance des cellules transformées donne alors naissance au processus tumoral. L'existence d'antioncogènes, gènes impliqués à l'opposé dans le vieillissement tissulaire, a été montrée par l'analyse d'hybrides somatiques ; certains antioncogènes ont récemment été isolés. Les interactions (neutralisantes ?) entre produits (protéines) de protooncogènes et d'antioncogènes font l'objet d'études qui débutent [10]. Le fait que le pouvoir transformant des RI implique des pertes d'antioncogènes montre que l'étude de la transformation par les RI doit permettre de caractériser de nouveaux antioncogènes. 
Les mécanismes de la tumorigenèse sont, cependant, plus subtils. En effet, la susceptibilité particulière à la survenue de cancers s'accompagne, en général, d'un manque de différenciation des fibroblastes [30]. L'histologie nous montre que ces cellules dérivant du mésoderme réalisent des associations étroites au sein de tous les organes avec les cellules épithéliales (réf. dans [30]). L'origine embryonnaire distincte des deux classes de cellules permet de supposer l'existence, entre chaque type cellulaire, de régulations de l'expression de protooncogènes et d'antioncogènes : façon d'exprimer la dynamique de nature dualiste de la croissance, de la différenciation et du vieillissement des organes. Les RI peuvent altérer ces réseaux de régulation à bien des niveaux dont certains se précisent peu à peu. Une vision cohérente des mécanismes de la cancérisation par les RI exige tout un ensemble de données supplémentaires. Les concepts nouveaux et les outils cellulaires et moléculaires dont nous disposons maintenant mettent cet objectif à notre portée.

\section{Remerciements}

Ce travail a bénéficié d'un soutien financier provenant du Comité de radioprotection d'EDF (contrat BOOL 34).

\section{BIBLIOGRAPHIE}

[1] ACADÉMIE DES SCIENCES - Cancérogenèse par les faibles doses de radiations ionisantes et normes de sécurité (Latarjet R., Ed.) Paris : Académie des sciences, 1988.

[2] ADAMSON E.D. - Oncogenes in development. Development, 1987, 99, 449-471.

[3] ATCHINSON M.L. - Enhancers : mechanisms of action and cell specificity. Ann. Rev. Cell. Biol., 1988, 4, 127-153.

[4] BISHOP W.R., BELL R.M. - Assembly of phospholipids into cellular membranes : biosynthesis, transmembrane movement and intracellular translocation. Ann. Rev. Cell. Biol., 1988, 4, 579-610.

[5] BOREK C. - Radiation oncogenesis in culture. Adv. Cancer Res., 1982, 32, 159-232.

[6] COX R., DEBENHAM P.C., MASSON W.K., WEBB M.B.T. - Ataxia telangectasia : a human mutation giving high-frequency misrepair of DNA double-stranded scissions. Mol. Biol. Med., 1986, 3, 229-244.

[7] FABRE F. - Induced recombinational activity is transmissible through mitotic divisions in yeast, (à paraître).

[8] FINCHAM R.S., OLIVER P. - Initiation of recombination. Nature, News and Views, 1989, 338, 14-15.

[9] GASSER S.M., LAEMMLI U.K. - A glimpse at the chromosomal order. Trends in Genetics, $1987,3,16-22$. 
[10] GREEN M.R. - When the products of oncogenes and antioncogenes meet. Cell, 1989, 56, 1-3.

[11] GROSOVSKY A.J., DE BOER J.G., DE LONG P.J., DROBETSKY E.A., GLICKMAN B.W. - Bases substitutions, frameshifts, and small deletions constitute ionizing radiation-induced point mutations in mammalian cells, Proc. Natl. Acad. Sci. USA, 1988, 85, 185-188.

[12] HANSEN M.F., CAVENEE W.K. - Tumor suppressors : recessive mutations that lead to cancer. Cell, 1988, 53, 172-173.

[13] HERSCHMAN H.R., BRANKOW D.W. - Ultraviolet irradiation transforms $\mathrm{C}_{3} \mathrm{H} / 10 \mathrm{~T} 1 / 2$ cells to a unique, suppressible phenotype. Science, 1986, 234, 1385-1387.

[14] HONG F.D., HUANG H.J.S., TO H., YOUNG L.J.S., ORO A., BOOKSTEIN R., LEE E.Y.H.P., LEE W.H. - Structure of the human retinoblastoma gene. Proc. Natt. Acad. Sci. USA, 1989, 86, 5502-5506.

[15] KANO Y., LITTLE J.B. - Mechanisms of human cell neoplastic transformation : $X$-ray-induced abnormal clone formation in long-term cultures of human diploid fibroblasts. Cancer Res., 1985, 45, 2550-2555.

[16] KLEIN G. - The approaching era of the tumor suppressor genes. Science, $1987,238,1539-1545$.

[17] LATARJET R., TUBIANA M. - The risks of induced carcinogenesis after irradiation at small doses. The uncertainties which remain after the 1988 UNSCEAR report. Int. J. Radiat. Oncol. Biol. Phys., 1989, 17, 237-240.

[18] LEE E.Y.P., TO H., SHEW J., BOOKSTEIN R., SCULLY P., LEE W.H. - Inactivation of the retinoblastoma susceptibility gene in human breast cancers. Science, 1988, 241, 218-221.

[19] LEMOTTE P.K., ADELSTEIN S.J., LITTLE J.B. - Malignant transformation induced by incorporated radionuclides in Balb/3T3 mouse embryo fibroblasts. Proc. Natl. Acad. Sci. USA, 1982, 79, 7763-7767.

[20] LITTLE J.B., NAGASAWA H., KENNEDY A.R. - DNA repair and malignant transformation : effect of $X$-irradiation, 12-0-tetradecanoyl-phorbol-13-acetate, and protease inhibitory on transformation and sister chromatid exchanges in mouse 10T1/2 cells. Radiat. Res., 1979, 79, 241-255.

[21] LOPEZ B., COPPEY J. - Promotion of double-strand break repair by human nuclear extracts preferentially involves recombination with intact homologous DNA. Nucleic Acids Res., 1987, 15, 6813-6826.

[22] LUMPKIN C.K., KEITH MCCLUNG J., PEREIRA-SMITH O.M., SMITH J.R. - Existence of high abundance antiproliferative mRNA's in senescent human diploid fibroblasts. Science, 1986, 232, 393-397.

[23] MAMURIS Z., PRIEUR M., DUTRILLAUX B., AURIAS A. - The chemotherapeutic drug melphalan induces breakage of chromosomes region rearranged in secondary leukemia. Cancer Genet. Cytogenet., 1989, 37, 65-77.

[24] MARTIN R.F., HASELTINE W.A. - Range of radiochemical damage to DNA with decay of iodine-125. Science, 1981, 213, 896-898. 
[25] McKINNON P.J. - Ataxia telangectasia : an inherited disorder of ionizingradiation sensitivity in man. Hum. Genet., 1987, 75, 197-208.

[26] MEHTA P.P., BERTRAM J.S., LOWENSTEIN W.R. - Growth inhibition of transformed cells correlates with their functional communication with normal cells. Cell, 1986, 44, 187-196.

[27] RADFORD I.R. - Evidence for a general relationship between the induced level of DNA double-strand breakage and cell killing after X-irradiation of mammalian cells. Int. J. Radiat. Biol., 1986, 49, 611-620.

[28] SAGER R. - Genetic suppression of tumor formation : a new frontier in cancer research. Cancer Res., 1986, 46, 1573-1580.

[29] SAKSELA O., RIFKIN D.B. - Cell associated plasminogen activation : regulation and physiological functions. Ann. Rev. Cell. Biol., 1988, 4, 93-126.

[30] SCHOR S.L., SCHOR A.M., HOWELL A., CROWTHER D. - Hypothesis : persistent expression of fetal phenotypic characteristics by fibroblasts is associated with an increased susceptibility to neoplastic disease. Expl. Cell. Biol., $1987,55,11-17$.

[31] SORRENTINO V., DROZDOFF V., ZEITZ L., FLEISSNER E. - increased radiation-induced transformation in $\mathrm{C} 3 \mathrm{H} / 10 \mathrm{~T} 1 / 2$ cells after transfer of an exogenous c-myc gene. Proc. Natl. Acad. Sci. USA, 1987, 84, 4131-4134.

[32] STEIN G.H., ATKINS L. - Membrane-associated inhibitor of DNA synthesis in senescent human diploid fibroblasts : characterization and comparison to quiescent cell inhibitor. Proc. Natl. Acad. Sci. USA, 1986, 83, 9030-9034.

[33] SU HUANG H.J., YEE J.K., SHEW J.Y., CHEN P.L., BOOKSTEIN R., FRIEDMAN T., LEE E.Y.P., LEE W.H. - Suppression of the neoplastic phenotype by replacement of the retinoblastoma gene in human cancer cells. Science, 1988, $242,1362-1366$.

[34] TAPPEL A.L. - Lipid peroxidation damage to cell components. Fed. Proc., $1973,32,1870-1874$.

[35] WEINTRAUB H. - Assembly and propagation of repressed and derepressed chromosomal states. Cell, 1985, 42, 705-711.

[36] WEISBROD S. - Active chromatin. Nature, 1982, 297, 289-295.

[37] WONG R.L., GUTOWSKI J.K., KATK M., GOLDFARB R.H., COHEN S. - Induction of ONA synthesis in isolated nuclei by cytoplasmic factors : inhibition by protease inhibitors. Proc. Natl. Acad. Sci USA, 1987, 84, 241-245. 